\title{
Reduced-basis output bound methods for heat transfer problems
}

\author{
D.V. Rovas, T. Leurent, C. Prud'homme, A.T. Patera * \\ Massachusetts Institute of Technology, Mechanical Engineering Department, Room 3-266, Cambridge, MA 02139, USA
}

\begin{abstract}
We describe a technique for the rapid and reliable prediction of outputs of interest, of elliptic partial differential equations with affine parameter dependence. To achieve efficiency, the reduced-basis method is used; reliability is obtained by the development of relevant a posteriori error estimators. We apply this method to the problem of designing a thermal fin, to effectively remove heat from a surface. A number of design parameters/inputs are considered. Each possible configuration, corresponding to different choices of the design parameters, needs to be evaluated by solving the heat conduction equation and calculating certain outputs of interest like the average temperature on the fin base.
\end{abstract}

Keywords: Reduced-basis method; A posteriori error estimation; Heat transfer

\section{Introduction}

In engineering and science, the use of numerical simulation is becoming increasingly important. The physical problems in consideration are often modeled by a set of partial differential equations and related boundary conditions; then, a discrete form of the mathematical problem is derived and a solution is obtained by numerical solution methods. As the physical problems become more complicated and the mathematical models more involved, current computational resources prove inadequate; the time required to perform the computation becomes unacceptably large. Especially in the field of optimization or design, where the evaluation of many different possible configurations is required - corresponding to different choices of the design parameters/inputs - reliable methods that reduce the complexity of the problem while at the same time preserve all relevant information, are becoming very important.

\section{Numerical method}

The method used in this paper is the reduced-basis output bound method developed in [1-4]; for details related to the implementation, see [2]. In designing new methods, certain qualities must be considered: efficiency, relevance and reliability.

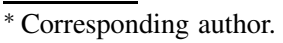

\subsection{Efficiency}

To achieve efficiency, we pursue the reduced-basis method; a weighted residual Galerkin-type method, where the solution is projected onto low-dimensional spaces with certain problem-specific approximation properties.The reduced-basis method has been proposed first by Nagy in [6], for the nonlinear analysis of structures. It has been further investigated and extended by Noor and Peters [7]. A priori theory has been developed by Fink and Rheinboldt [10], Porsching [11] and Barret and Redien [9].

\subsection{Relevance}

Usually in a design or optimization procedure, we are not interested in the field solution or norms of it, but rather in certain design measures, such as the drag coefficient in the case of flow past a bluff body, or the average temperature on a surface in the case of heat conduction. The methods considered give accurate approximations to these outputs of interest, defined as functional outputs of the field solution.

\subsection{Reliability}

To quantify the error introduced by the reduced-basis method, a posteriori error analysis techniques must be invoked. There has recently been much interest in methods for a posteriori error estimation, especially to estimate the 
discretization error for the finite element method; a review can be found in [8]. Most error estimators developed give bounds for abstract norms of the error. A posteriori error estimators for outputs of interest have been developed in the reduced-basis context; for more details see [3].

Special affine parameter dependence of the differential operator is exploited to develop a two-stage offline/online blackbox computational method. In the online stage, for every new set of design parameters, an approximation to the output of interest and an associated error bound is calculated. The computational complexity of the online stage of the procedure scales only with the dimension of the reduced-basis space (which is usually $O(10)$ ) and the parametric complexity of the partial differential operator; for more details see [2]. Solution of the governing equations in 'real-time', can thus be achieved.

\section{Thermal fin problem}

In this example, we consider a three-dimensional thermal fin used to effectively remove heat from a surface. The three-dimensional fin, shown in Fig. 1, consists of a vertical central 'post' and four horizontal 'subfins'; the fin conducts heat from a prescribed uniform flux 'source' at the root, through the large-surface-area subfins to surrounding flowing air.

The fin is characterized by a seven-component parameter vector, $\mu=\left(\mu^{1}, \ldots, \mu^{7}\right)$, where $\mu^{i}=k^{i}, i=1, \ldots, 4$; $\mu^{5}=\mathrm{Bi} ; \mu^{6}=L$; and $\mu^{7}=t ; \mu$ may take on any value in a specified design space $\mathcal{D} \subset \mathbb{R}^{7}$. Here $k^{i}$ is the thermal conductivity of the $i$-th subfin (normalized relative to the post conductivity $k^{0} \equiv 1$ ); $\mathrm{Bi}$ is the Biot number, a nondimensional heat transfer coefficient reflecting convective transport to the air at the fin surfaces; and $L$ and $t$ are the length and thickness of the subfins (normalized relative to

\section{Bi: heat transfer coefficient}

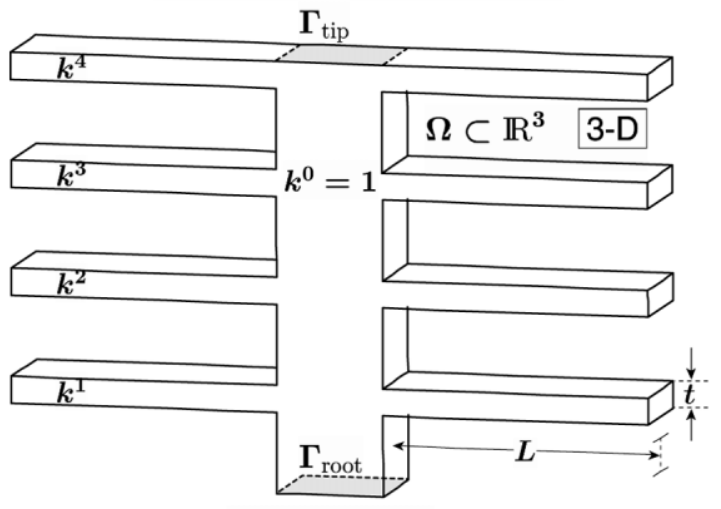

Root: Heat In

Fig. 1. 3D thermal fin. the post width). The fin is one unit deep (the root is square) and four units tall.

We consider several outputs of interest. The first output, $T_{\text {root }} \in \mathbb{R}$, is taken to be the average temperature of the fin root normalized by the prescribed heat flux into the fin root. This output relates directly to the cooling efficiency of the fin - lower values of $T_{\text {root }}$ imply better performance. Another output is the volume of the fin, which represents weight and material cost - thus lower values are preferred. In order to optimize the design, we must be able to rapidly evaluate $T_{\text {root }}(\mu)$ and the volume of the fin $\mathcal{V}$ for a large number of parameter values $\mu \in \mathcal{D}$.

The steady-state temperature distribution within the fin, $u(\mathbf{x})$, is governed by the elliptic partial differential equation

$-k^{i} \nabla^{2} u^{i}=0$ in $\Omega^{i}, \quad i=0, \ldots, 4$,

where $\nabla^{2}$ is the Laplacian operator, and $u^{i}$ refers to the restriction of $u$ to $\Omega^{i}$. Here $\Omega^{i}$ is the region of the fin with conductivity $k^{i} ; i=0, \ldots, 4: \Omega^{0}$ is thus the central post, and $\Omega^{i} ; i=1, \ldots, 4$, corresponds to the four subfins. We must also ensure continuity of temperature and heat flux at the conductivity-discontinuity interfaces $\Gamma^{i} \equiv \partial \Omega^{0} \cap \partial \Omega^{i}$, $i=1, \ldots, 4$, where $\partial \Omega^{i}$ denotes the boundary of $\Omega^{i}$ :

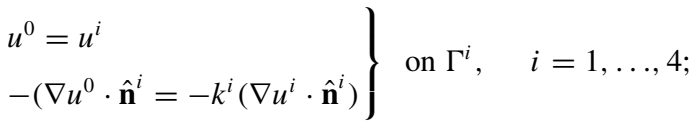

here $\hat{\mathbf{n}}^{i}$ is the outward normal on $\partial \Omega^{i}$. Finally, we introduce a Neumann flux boundary condition on the fin root

$-\left(\nabla u^{0} \cdot \hat{\mathbf{n}}^{0}\right)=-1$ on $\Gamma_{\text {root }}$,

which models the heat source; and a Robin boundary condition

$-k^{i}\left(\nabla u^{i} \cdot \hat{\mathbf{n}}^{i}\right)=\operatorname{Bi} u^{i}$ on $\Gamma_{\mathrm{ext}}^{i}, \quad i=0, \ldots, 4$,

which models the convective heat losses. Here $\Gamma_{\text {ext }}^{i}$ is that part of the boundary of $\Omega^{i}$ exposed to the fluid that is $\partial \Omega \backslash \Gamma_{\text {root }}$.

For every choice of the design parameter-vector $\mu-$ which determines the $k^{i}, \mathrm{Bi}$, and also the fin geometry through $L$ and $t-$ solution of the above system of equations yields the temperature distribution $u(\mathbf{x} ; \mu)$ in the fin. The output of interest, $T_{\text {root }}(\mu)$, can be expressed as $T_{\text {root }}(\mu)=\ell_{\text {root }}^{O}(u(\mathbf{x} ; \mu))$, where

$\ell_{\text {root }}^{O}(v)=\int_{\Gamma_{\text {root }}} v$

( $\Gamma_{\text {root }}$ is of area unity). As for the volume, it is given by the following formula

$\mathcal{V}=4+8 L t$. 


\section{Results}

In multi-criterion optimization, we consider various (competing) outputs of interest, say volume, $\mathcal{V}$, and root temperature, $T_{\text {root }}$. Changing the dimensions of the fin by selecting different $L$ and $t$ will (say) decrease the volume of the fin, and hence material requirements - but also (typically) increase the fin base temperature. It is thus of interest to determine all possible operating points, that is, to generate the map of the 'achievable set'. In general, this will be prohibitively expensive unless one has recourse to a very low-dimensional representation, such as the reducedbasis approximation.

We consider this problem for constant conductivities $k_{i}=1, i=0, \ldots, 4$, and Biot number $\mathrm{Bi}=0.1$. We then select 100 points in the two dimensional design space $[t, L]=[0.2,0.4] \times[2.0,3.0]$ and evaluate our bounds for $T_{\text {root }}$ with an error tolerance of $1 \%$. Since in this design we wish to be sure that the actual temperature will be less than our prediction, we choose to construct our map based on the upper bound obtained by the error estimator. We are thus insured that at each design point, the actual temperature will be lower than that on our curve.

Each evaluation produces a point on the $T_{\text {root }}-\mathcal{V}$ plane, thus generating the achievable set. Obvious optimality conditions require that we remain on the left or lower boundaries of the achievable set, known as the efficient frontier or trade-off curve in Pareto analysis. As we can see from Fig. 2, we can decrease the volume with relatively small

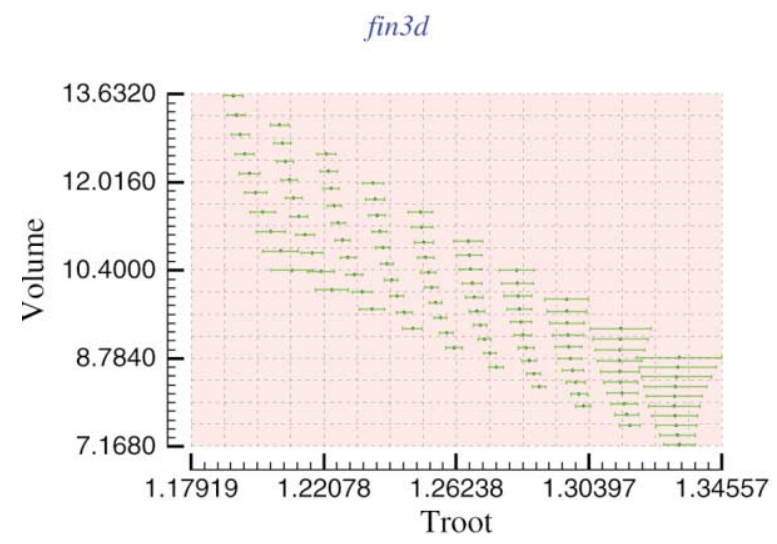

Fig. 2. Achievable set. increase in temperature up to the point were the left and lower boundaries cross; after that a small further possible volume reduction results in a steep rise in base temperature.

\section{Acknowledgements}

This work was supported by the Singapore-MIT Alliance, by AFOSR Grant F49620-97-1-0052, and by NASA Grant NAG1-1978.

\section{References}

[1] Machiels L, Maday Y, Oliveira IB, Patera AT, Rovas DV. Output bounds for reduced-basis approximations of symmetric positive definite eigenvalue problems. C.R. Acad Sci Paris, Série I, to appear.

[2] Maday Y, Machiels L, Patera AT, Rovas DV. Blackbox reduced-basis output bound methods for shape optimization. In Proceedings 12th International Domain Decomposition Conference, Chiba, Japan, 2000.

[3] Patera AT, Rovas DV, Machiels L. Reduced-Basis OutputBound Methods for Elliptic Partial Differential Equations. SIAG/OPT Newsletter Aug 2000;11(2):4—9.

[4] Maday Y, Patera AT, Rovas DV. A blackbox reducedbasis output bound method for noncoercive linear problems. MIT-FML Report 00-2-1, 2000; also in the Collège de France Series, to appear.

[5] Maday Y, Patera AT, Peraire J. A general formulation for a posteriori bounds for output functionals of partial differential equations; application to the eigenvalue problem. C.R. Acad. Sci. Paris, Série I, 1999;328:823-829.

[6] Nagy DA. Model representation of geometrically nonlinear behavior by the finite element method. Comput Struct 1977;10:683-688.

[7] Noor AK, Peters JM. Reduced basis technique for nonlinear analysis of structures. AIAA J 1980;18(4):455-462.

[8] Ainsworth M, Oden JT. A Posteriori Error Estimation in Finite Element Analysis. John Wiley and Sons, January, 2000.

[9] Barret A, Redien G. On the reduced basis method. Z Angew Math Mech 1995;75(7);543-549.

[10] Fink JP, Rheinboldt WC. On the error behavior of the reduced basis technique in nonlinear finite element approximations. Z Angew Math Mech 1983;63:21-28.

[11] Porsching TA. Estimation of the error in the reduced basis method solution of nonlinear equations. Math Comp 1985;45(172):487-496. 